\title{
Fabrice Boudjaaba (dir.), Le Travail et la famille en
} milieu rural. $\mathrm{XVI}^{e}$-XXI ${ }^{e}$ siècle

Rennes, Presses universitaires de Rennes, coll. « Histoire », 2014

Jérôme Bourdieu

\section{CpenEdition}

\section{Journals}

Édition électronique

URL : http://journals.openedition.org/travailemploi/7189

DOI : 10.4000/travailemploi.7189

ISSN : 1775-416X

Éditeur

DARES - Ministère du Travail

Édition imprimée

Date de publication : 1 juillet 2016

Pagination : 125-128

ISSN : 0224-4365

Référence électronique

Jérôme Bourdieu, « Fabrice Boudjaaba (dir.), Le Travail et la famille en milieu rural. xv1 ${ }^{e}-x x l^{e}$ siècle », Travail et Emploi [En ligne], 147 | juillet-septembre 2016, mis en ligne le 11 juillet 2019, consulté le 25 septembre 2020. URL : http://journals.openedition.org/travailemploi/7189 ; DOI : https://doi.org/ 10.4000/travailemploi.7189 


\section{NoteS DE LECTURE}

\section{Le Travail et la famille en milieu rural. $\mathrm{XVI}^{\mathrm{e}}-\mathrm{XXI}^{\mathrm{e}}$ siècle}

Fabrice Boudjaaba (dir.)

Rennes, Presses universitaires de Rennes, coll. « Histoire », 2014, 281 p.

Lu par Jérôme Bourdieu*

Ce livre, aboutissement d'un travail collectif coordonné par Fabrice Boudjaaba, rassemble des historiens et des sociologues français qui, à partir d'études de cas précis, proposent d'aborder sous un angle nouveau le poids des structures familiales dans le monde rural entre le XVI et le XXI siècle. À force de mesurer le rôle et le fonctionnement de la famille à l' aune de la transmission au moment du décès, de construire des catégories trop inspirées par une vision juridique et d'établir des typologies fondées sur l'opposition entre partage inégalitaire et égalitaire de l'héritage, une part importante de l'historiographie, sans doute également par un effet de sources, a contribué à effacer des modes de fonctionnements plus ordinaires de la famille. Il s'agit de ceux qui impliquent les vivants, tout au long du cycle de vie, dans des échanges n'engageant pas en une fois tout le capital de la famille. En particulier, comme le met en avant Fabrice Boudjaaba dans son introduction, tout ce qui concerne le travail et son organisation au sein de la famille, tout ce qui mobilise l'ensemble des membres de la famille notamment dans le cadre de l'exploitation agricole (mais cela vaudrait sans doute aussi dans le cadre d'activités artisanales ou commerciales), tout ce qui a trait à l'accès au travail et, en premier lieu à la formation, a longtemps ainsi été passé sous silence. Sur cette base, l'ouvrage se fixe comme « objectif majeur » d' ' interroger l'articulation et les concurrences entre une définition du travail comme ressource collective au service de l'exploitation et du groupe domestique et une définition du travail comme ressource individuelle permettant de construire son propre parcours professionnel, familial et patrimonial » (p. 18).

Pour y parvenir, l'ouvrage opère en trois temps. La première partie, la plus ample, porte sur la manière dont le travail est organisé par la famille et, parfois, lui échappe. La deuxième aborde la question plus spécifique de la formation, de l'éducation et de l'accès au travail. La dernière enfin, plus brève, examine la capacité de la famille à produire de la mobilité sociale, à moins qu'il ne faille plutôt dire qu'elle se propose d'analyser comment une mobilité sociale hors du monde rural est possible malgré la famille.

\footnotetext{
* Paris School of Economics (PSE) ; Institut national de recherche agronomique (Inra) ; École des hautes études en sciences sociales (EHESS).
} 
Les textes qui constituent la première partie ont pour trait prédominant de faire une place centrale aux effets de structure. Le chapitre de Matteo di Tullio consacré à la Lombardie du XVI ${ }^{e}$ siècle analyse le lien, d'une part, entre la taille des ménages et les structures démographiques et, d'autre part, les «contextes agricoles », les types de productions, les formes de propriétés foncières, la disponibilité des terres. La présence inégale du famulato ${ }^{1}$ dans les familles de la plaine et dans celles des collines en est une manifestation importante. Les stocks agricoles domestiques et leur inégale répartition entre les ménages, dont l'auteur trouve une mesure grâce aux recensements de population, fournissent un autre élément permettant de contraster les pratiques familiales en fonction des contextes productifs. Dans le même ordre d'idées, les pratiques d'association et d'indivision que Fabrice Boudjaaba et Francisco García Gonzáles observent au XVIII siècle, l'un en Normandie, l'autre dans la Sierra de Alcaraz en Espagne, apparaissent comme « une forme transitoire de propriété adaptée au besoin et aux faibles capacités d'investissement des jeunes ménages » (p. 84), un trait fréquent de ces deux sociétés rurales. De même, examinant les fratries comme terrain de solidarités familiales possibles, Laurent Herment constate que les prêts entre frères et sœurs restent exceptionnels et que les seuls cas de mise en commun de ressources correspondent à des associations, elles-mêmes très rares chez les agriculteurs.

La deuxième partie porte sur la confrontation entre les logiques familiales d'une part, et celles de formation et d'accès à une éducation offerte par des institutions extérieures à la famille d'autre part. Ainsi, le choix d'envoyer les enfants à l'école se révèle guidé non pas seulement par des critères économiques mais aussi par un sentiment d'obligation morale. Mais ce dernier se heurte au fait que l'accès à l'école inculque des aspirations nouvelles à ceux qui la fréquentent. C'est le cas en particulier pour les filles, malgré les efforts constants pour leur y assigner une place différente de celle des garçons. L'article de Carmen Sarasúa consacré à la scolarisation des enfants en Espagne au XIX ${ }^{e}$ siècle et à la diversité des modes de financement des écoles, comme celui de Jean-Louis Escudier qui montre comment l'enseignement agricole ménager est un moyen de dissuader les filles de reprendre l'activité de leur père dans la viticulture en France au Xx ${ }^{\mathrm{e}}$ siècle, illustre cette ambivalence.

La troisième partie (à laquelle aurait pu se rattacher le chapitre de Christophe Giraud sur l'hétérogamie parentale chez les agriculteurs en France à partir des années 1970) peut se lire comme un examen des conséquences des deux précédentes. Elle fait apparaître que la mobilité sociale, loin d'être réglée de manière un peu mécanique par des normes de transmission au moment de l'héritage, est tributaire, d'une part, d'un travail des familles qui dure tout au long de la vie dans une tension parfois forte entre les générations - comme le montrent tant Hervé Bennezon que Céline Bessière et Sibylle Gollac - et, d'autre part, des effets socialement différenciés du contexte économique et de ses transformations. Le cas des destins paysans de la Vénétie au Xx ${ }^{e}$ siècle qu'aborde

1. Un famulato est un " "familier" qui effectue des corvées domestique ou agricole auprès des familles ». Ils en deviennent ainsi « membres sans être pour autant liés par liens du sang » (p. 46). 
David Celetti établit ainsi que ceux qui parviennent à maintenir leurs exploitations, au prix de grands sacrifices et, in fine, non sans regrets, sont des paysans moyens alors que les petits n'ont pas survécu et que les plus grands propriétaires ont réalisé des reconversions réussies hors de l'agriculture.

D'autres entrées auraient pu être choisies pour la présentation de l'ouvrage ; on va en proposer deux exemples pour illustrer combien ce livre se prête à des angles de lecture divers et féconds. La première entrée est l'articulation entre les activités agricoles et les autres activités rurales, articulation qui est largement placée sous contrôle familial : ce thème traverse plusieurs chapitres, notamment celui qui est écrit par Frank Dellion sur l'usine de la Schappe. Il est très présent aussi dans celui de Laurent Herment sur l'endettement et les collaborations intrafamiliales, ou encore dans celui de David Celetti sur l'enfermement dans l'agriculture que s'imposent les cultivateurs d'exploitations de taille moyenne. Une seconde entrée possible est celle de la situation des femmes et du rapport, différent de celui des hommes, qu'elles entretiennent et, souvent, qu'elles sont forcées d'entretenir vis-à-vis de chacun des fonctionnements examinés dans le livre. Le contraste est évident pour les femmes de marins dans la Bretagne du XVIII ${ }^{e}$ siècle qu'aborde Emmanuelle Charpentier : ici, la division du travail au sein des couples est portée à sa limite. Mais ce contraste entre hommes et femmes n'est pas moins important dans la participation aux activés non agricoles que l'on observe dans le cas de l'usine de la Schappe analysé par Frank Dellion. Et il s'exprime aussi très tôt dans le cycle de vie, dès l'accès à des apprentissages et à des formations fortement différenciées que décrit Carmen Sarasúa dans l'enseignement primaire en Espagne au XIX ${ }^{\mathrm{e}}$ siècle. Le contraste se manifeste enfin par une exclusion de principe des filles de certains métiers. Étudiant la viticulture en France au XIX ${ }^{\mathrm{e}}$ siècle, Jean-Luc Escudier parle ainsi de «non-transmission d'un métier» (p. 151) (dans ce cas, l'exclusion des femmes est institutionnalisée au niveau national par le fait qu'il n'existe aucun établissement d'enseignement viticole ouvert aux filles). La mise à l'écart des filles s'observe encore dans l'analyse que propose Frédérique El Amrani des trajectoires professionnelles des jeunes filles de l'Anjou rural entre 1920 et 1950. Alors qu'elles rêvent d'émancipation, elles sont assignées à des positions et à des rôles féminins par leur famille, et souvent par leur mère.

Peut-être aurait-il été utile que les catégories utilisées (famille, travail, solidarité, rural, agricole, etc.) soient plus souvent soumises à une lecture critique et historique, non pas pour poser des définitions rigides a priori, mais parce que la définition même de ces catégories est en jeu dans les phénomènes observés. La famille fonctionne ainsi sous différentes modalités : dans la logique de l'héritage, elle est, plus qu' ailleurs peutêtre, une catégorie juridique ; dans le cas de l'entraide quotidienne, elle se joue entre ses membres géographiquement proches ; quand il est question de prêt, d'association, de migration ou de placement professionnel, ce sont encore d'autres définitions de la famille qui sont activées. Et apparaissent clairement les variations dans l'espace et dans le temps de ce qui fait de chacune de ces modalités de fonctionnement de la famille une ressource économique et sociale. L'importance de la question de la corésidence, 
qui préoccupe tant les historiens depuis que la famille nucléaire a été établie comme la norme ordinaire, parait devoir être relativisée si l'on pense que la question du partage effectif d'un lieu d'habitation commun n'en est qu'un aspect, sans doute en lui-même susceptible d'interprétations diverses. D'une part, la cohabitation urbaine dans des pièces exiguës a peu de choses à voir avec la cohabitation dans des fermes disposant de plusieurs bâtiments. D'autre part, son importance s'avère relative si on la met en regard de l'entraide ordinaire qui se joue entre proches ${ }^{2}$ (il reste que, pour des raisons de sources au moins, ces formes ordinaires de l'entraide restent plus difficiles à appréhender). Dans un autre registre, la question de la nature du travail (du travail agricole notamment et de sa différenciation effective avec d'autres types de travaux) mais aussi celle de la définition et de la reconnaissance du travail féminin, ainsi que - le thème n'est pas abordé - celle du travail des enfants se posent lorsqu'on s'interroge sur le rôle de la famille sur l'organisation collective du travail. On peut relever pour finir que le concept de solidarité présente lui aussi une polysémie qui lui donne force d'interprétations mais qui pourrait conduire à des constructions plus structurées dans l'ouvrage : en particulier, on sent des tensions dans le concept de solidarité familiale entre un altruisme inscrit sur le mode du « cela va de soi » dans les comportements individuels, et la possibilité de solidarités à la fois contraintes et contraignantes que les uns sollicitent, que d'autres utilisent et que certains veulent fuir.

Ce livre, par le déplacement de focale qu'il propose, témoigne assurément de l'ampleur des travaux à mener sur des terrains que l'on aurait pu penser surinvestis.

2. Proches à la fois par la faible distance géographique et par les liens de parenté. 